\title{
MOCVD-GROWN InGaP/GaAs EMITTER DELTA DOPING HETEROJUNCTION BIPOLAR TRANSISTORS
}

\author{
K. F. YARN* \\ Far East College, Department of Electrical Engineering, Optoelectronic Semiconductor Center, \\ Hsin-Shih, Tainan, Taiwan 744, Republic of China
}

(Received 17 December 2001; In final form 21 March 2002)

\begin{abstract}
The influence of delta doping sheet at base-emitter (BE) junction for an $\mathrm{InGaP} / \mathrm{GaAs}$ heterojunction bipolar transistor (HBT) with a $75 \AA$ undoped spacer layer is investigated. A common emitter current gain of 235 , an offset voltage as small as $50 \mathrm{mV}$ and an Ic ideal factor of 1.01 are obtained, respectively. The use of delta doping sheet at BE junction results in a high gain and low offset voltage HBT. The improvement of current gain and offset voltage may be attributed to the reduction of BE potential spike by introducing a delta doping layer even without the BE junction passivation.
\end{abstract}

Keywords: Delta doping; Heterojunction bipolar transistor

\section{INTRODUCTION}

Heterojunction bipolar transistors (HBT) with AlGaAs/GaAs material have attracted considerable attention in microwave and digital application due to their high current handling capability and high speed [1]. The use of wide emitter bandgap introduces a band discontinuity $\Delta \mathrm{Ev}$ at the valence which suppresses the hole current injection from base to emitter. Thus, the current gain can be amplified about the order of $\exp \left(\Delta E_{v} / E_{g}\right)$ or a higher BE doping ratio is permitted due to the hole confinement. The increase of base doping level also reduces the base resistance that is very important in high frequency operation. However, there still exist some drawbacks and tradeoff associated with the potential spike resulting from the increase of conduction band discontinuity $\Delta E_{c}$ while the $\Delta E_{v}$ is increased.

Recently, many researchers have focused on the $\operatorname{In}_{0.49} \mathrm{Ga}_{0.51} \mathrm{P} / \mathrm{GaAs}$ aluminum-free and lattice-match material system [2]. There are the following advantages in using $\mathrm{InGaP} / \mathrm{GaAs}$ GaAs compared with AlGaAs/GaAs: (1) a higher $\Delta \mathrm{E}_{v} / \Delta \mathrm{E}_{c}$ ratio (2) a higher etching selectivity between InGaP and GaAs interface (3) a lower surface recombination velocity (4) a lower DX center density. The good performance of $\mathrm{InGaP} / \mathrm{GaAs}$ related device implies the potential for future circuit applications [3]. Several methods have been considered to solve the problem of increased CE offset voltage $\Delta V_{\mathrm{CE}}$. Grinberg et al. [2] have proposed the concept of graded EB junction, which however, introduces the additional complexities

* Present Address: P. O. Box 345, Tainan, Taiwan 704, Republic of China

ISSN 0882-7516 print; ISSN 1563-5031 online (C) 2002 Taylor \& Francis Ltd

DOI: $10.1080 / 0882751021000012544$ 
and difficulties needed to the control of the graded layer during epitaxial growth. The main purpose of this study is to obtain a new method to effectively reduce the potential spike, while a high current gain at low collector region is simultaneously maintained. Two types of structures (sample A and B) with spacer layer are investigated, the difference between $\mathrm{A}$ and $\mathrm{B}$ is that sample $\mathrm{A}$ has a delta doping layer inserted at the EB junction. By using the delta-doped technique, the electron spike barrier is reduced while the effective hole barrier is still increased. Thus, the total ratio of spike barrier $\Delta E_{v} / \Delta E_{c}$ is enhanced. This implies an InGaP/GaAs HBT with a low $\mathrm{CE}$ offset voltage $\Delta \mathrm{V}_{\mathrm{CE}}$ and a high current gain can be achieved.

\section{DEVICE STRUCTURE AND FABRICATION}

The cross section of the fabricated device (sample A) grown by AIXTRON 2400 metal organic chemical vapor deposition (MOCVD) system is schematically shown in Figure 1. Epitaxial layers were subsequently grown on the (100)-oriented $n^{+}$-GaAs substrate. $\mathrm{Si}_{2} \mathrm{H}_{6}$, $\mathrm{CBr}_{4}$ and $\mathrm{DCpMg}$ were used as $n$ - and $p$-type dopants, respectively. The delta-doped $n^{+}$ layer with a high doping level of $1 \times 10^{12} \mathrm{~cm}^{-2}$ was inserted between the spacer ( $i$-GaAs) and emitter. In order to compare the current-voltage $(I-V)$ characteristics, InGaP/GaAs HBT without delta-doped layer (sample B) is also included.

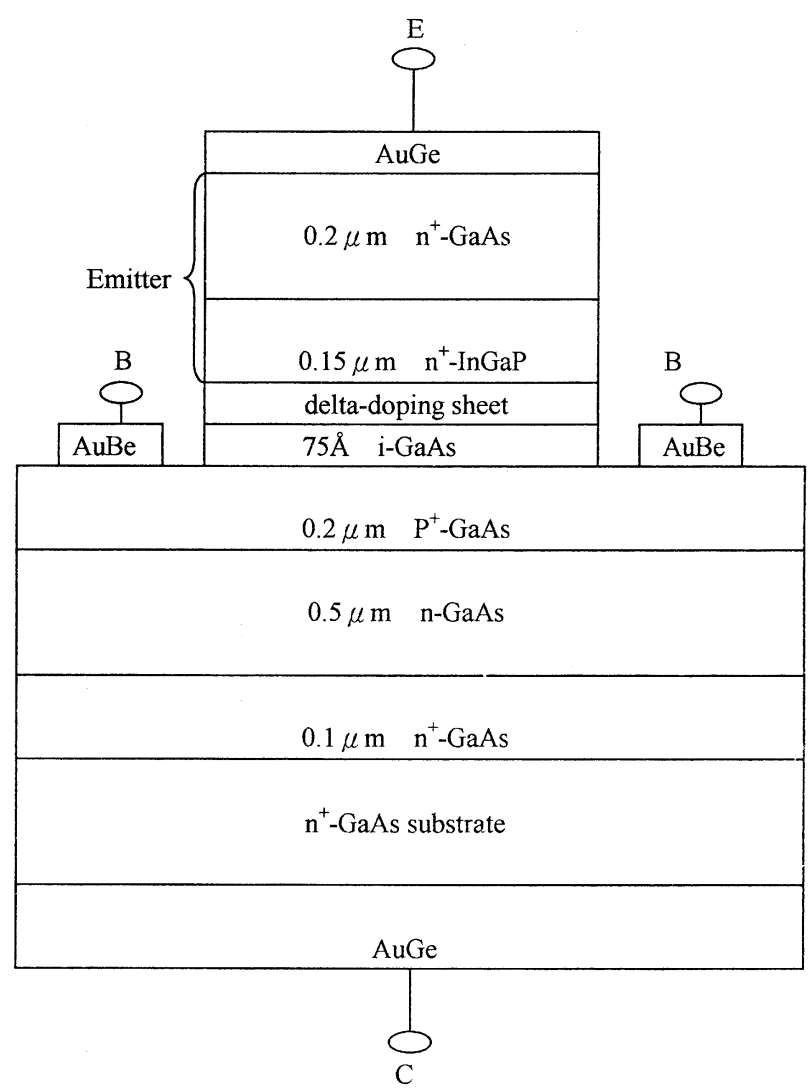

FIGURE 1 Schematic cross-section of delta-doped InGaP/GaAs HBT structure. 
Deposited on the substrate is an $n^{+}$-GaAs buffer of $0.1 \mu \mathrm{m}$ thickness with a doping level of $1 \times 10^{18} \mathrm{~cm}^{-3}$. Then, about $0.5 \mu \mathrm{m}$ of $n$-GaAs collector with a doping concentration of $5 \times 10^{16} \mathrm{~cm}^{-3}$ was grown, followed by a $0.2 \mu \mathrm{m}$ thick $p^{+}$-GaAs $\left(p=1 \times 10^{19} \mathrm{~cm}^{-3}\right)$ base. The emitter layer consisted of a $75 \AA$ undoped GaAs spacer, a delta-doped $n^{+}$layer, a $0.15 \mu \mathrm{m}$-thick $n^{+}$-InGaP $\left(n=5 \times 10^{17} \mathrm{~cm}^{-3}\right)$ and finally a $0.2 \mu \mathrm{m}$-thick $n^{+}$-GaAs $\left(n=1 \times 10^{18} \mathrm{~cm}^{-3}\right)$ cap layer was deposited. Mesa etching with a $\mathrm{H}_{2} \mathrm{SO}_{4}: \mathrm{H}_{2} \mathrm{O}_{2} 60 \mathrm{H}_{2} \mathrm{O}$ solution was used to define the device. $\mathrm{A} \mathrm{NH}_{4} \mathrm{OH}: \mathrm{H}_{2} \mathrm{O}_{2}: 3 \mathrm{H}_{2} \mathrm{O}$ solution was used to remove the $\mathrm{GaAs}$ top layer and then a $\mathrm{HCl}: \mathrm{H}_{2} \mathrm{O}$ solution with an etching rate of $25 \AA / \mathrm{sec}$ was used to etch the InGaP layer and exposed the base region. $\mathrm{Au} / \mathrm{Be}$ was employed for the $p^{+}-\mathrm{GaAs}$ base contact. The emitter and the collector of the device, were both metallized with $\mathrm{Au} / \mathrm{Ge}$. The emitter area is $80 \mu \mathrm{m} \times 80 \mu \mathrm{m}$ and the collector-to-emitter area ratio is 20 . The DC $I-V$ characteristics of the devices were measured at room temperature by HP $4145 \mathrm{~B}$.

\section{RESULTS AND DISCUSSION}

In order to enhance the performance of HBT, the EB potential spike plays an important factor to determine the $\mathrm{CE}$ offset voltage $\Delta V_{\mathrm{CE}}$ [4]. Under the external bias, the potential spike of HBT with (sample A) and without (Sample B) a delta-doped sheet are investigated. The magnitudes of EB potential spikes are calculated by solving the Poissons' equation. The potential spike of sample $\mathrm{B}$ is about $195 \mathrm{mV}$ at $V_{\mathrm{BE}}=1.0 \mathrm{~V}$, but that of sample A is negligible. The $\mathrm{EB}$ potential spike is nearly pulled down by the insertion of a delta-doped sheet.

The experimental Gummel plots of the studied device (sample A) with a delta-doped sheet are shown in Figure 2. The Ic ideal factor of 1.01 is near to unity, which is attributed to the elimination of the potential spike at the EB junction. On the other hand, the ideal factor of base current is 1.24 that is dominated by four components in base current: (1) injection current from base to emitter (2) recombination current in the neutral base (3) surface recombination current in the base (4) recombination current in the BE depletion region. It is obvious that the last three components are more important than the first component due to the large band discontinuity $\Delta E_{v}$ at EB junction. A previous report [5] shows that the space charge recombination current dominates the base current.

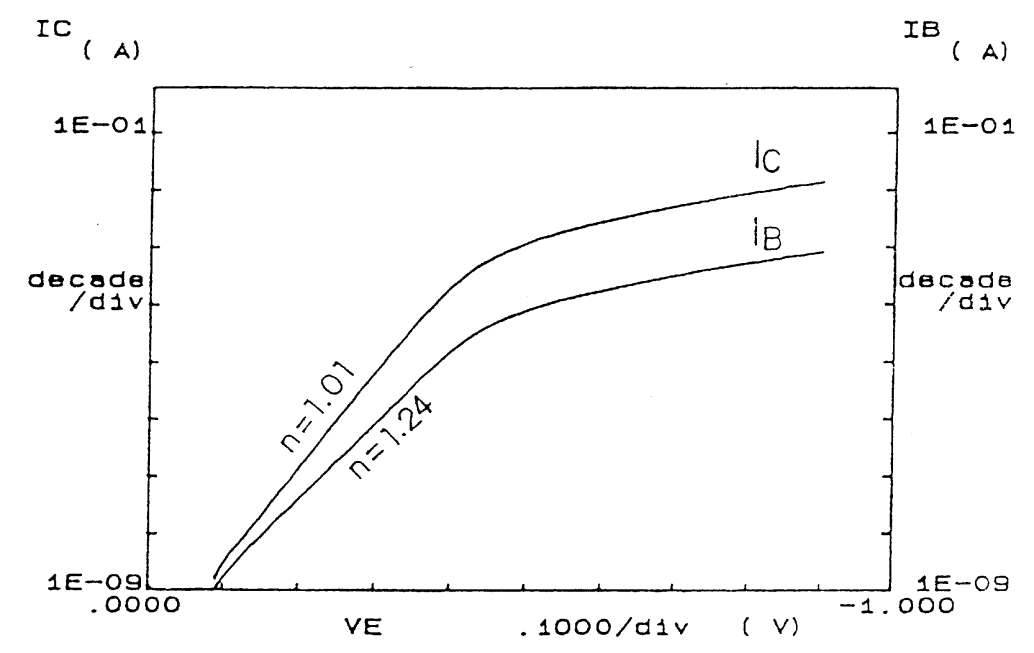

FIGURE 2 The ideal factor of collector and base current are 1.01 and 1.24, respectively. 
IC

$(\mathrm{mA})$

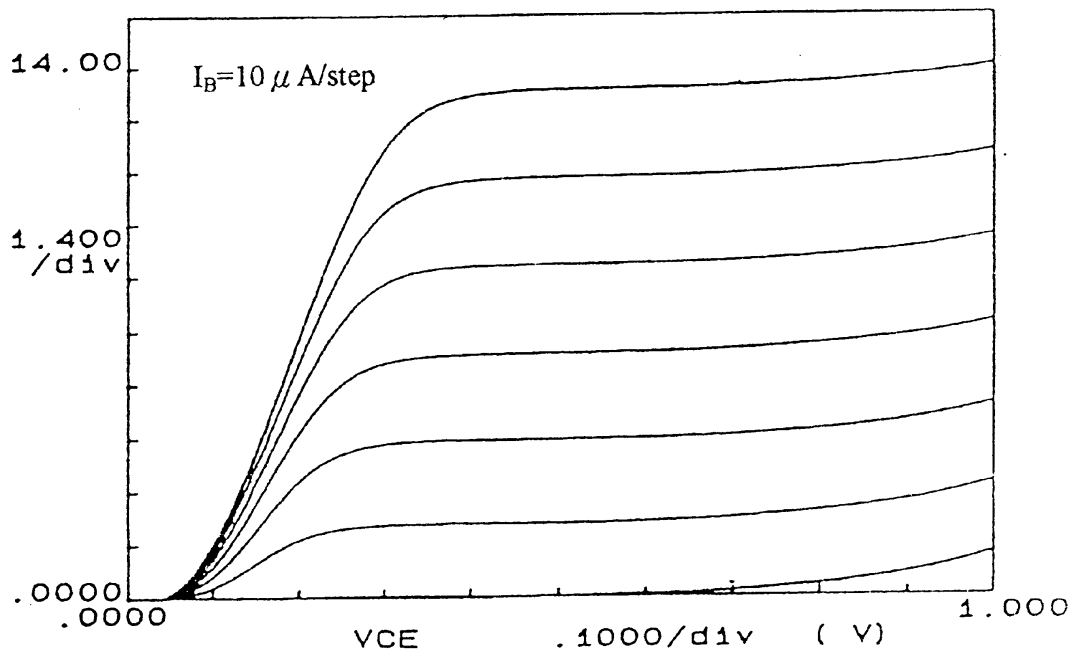

FIGURE 3 The typical common-emitter I-V characteristic of the delta-doped InGaP/GaAs HBT.

The electrical property of the common-emitter current-voltage $(I-V)$ curve of sample A is shown in Figure 3. The maximum common-emitter current gain of 235 at $I_{c}=14 \mathrm{~mA}$ is better than that of studied conventional HBT $(\beta=120)$ (sample B) and double $\operatorname{HBT}(\beta=150)$ without the delta doping structure based on the InGaP/GaAs material system [6].

In addition, it is noteworthy that the offset voltage $\Delta V_{\mathrm{CE}}$ is only $50 \mathrm{mV}$ for sample $\mathrm{A}$ and this value is shown in Figure 4. This small offset voltage is obtained even without using grading or passivation structure and is due to the suppression of the EB potential spike by using the spacer and delta doping layers.

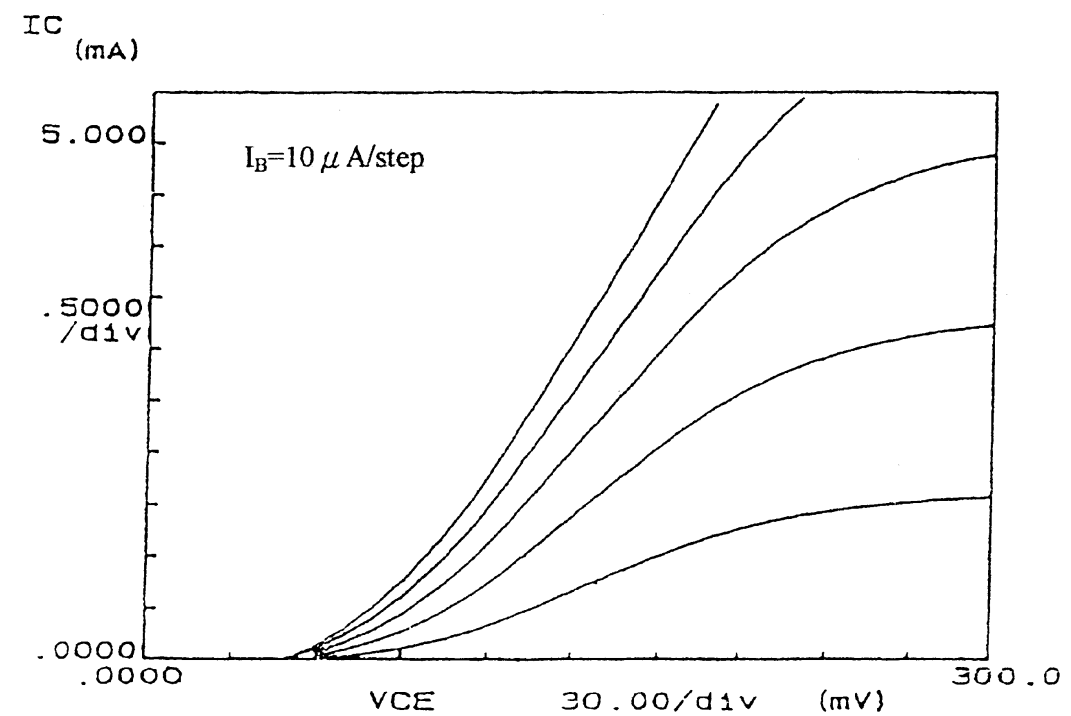

FIGURE 4 The offset voltage as low as $50 \mathrm{mV}$ in common emitter configuration shown in low voltage range. 


\section{SUMMARY}

An improved delta-doped InGaP/GaAs HBT with a $75 \AA$ spacer layer is fabricated successfully. The use of delta doping sheet located at BE interface makes the potential spike negligible significantly. In this study, a common-emitter current gain of 235 with an offset voltage as low as $50 \mathrm{mV}$ is achieved at the base thickness of $0.2 \mu \mathrm{m}$. The ideal factors of $I_{c}$ and $I_{B}$ are 1.01 and 1.24, respectively. Based on the idea of adding the delta doping sheet to suppress the BE potential spike, a high current gain and low offset voltage HBT will be developed by reducing the base thickness.

\section{Acknowledgement}

I am pleased to express my appreciation to those who helped with the growth of the epilayers, the technical assistance and typewriting, especially Miss C. C. Wu.

\section{References}

[1] Asbeck, P. M., Chang, M. F., Wang, K. C., Miller, D. L., Sullivan, G. J., Sheng, N. H., Sovero, E. and Higgins, J. A. (1987). IEEE Trans. Electron Dev., 34, 2571.

[2] Grinberg, A. A., Shur, M. S., Fisher, R. J. and Morkoc, H. (1984). IEEE Trans. Electron Dev., 31, 1758.

[3] Wu, C. C. and Lu, S. L. (1992). IEEE Electron Dev. Lett., 13, 418.

[4] Lee, S. C., Kuo, J. N. and Lin, H. H. (1984). Appl. Phys. Lett., 45, 1114.

[5] Liu, W. and Harris, J. S. (1992). IEEE Trans. Electron Dev., 39, 2726.

[6] Yang, Y. F., Hsu, C. C. and Yang, E. S. (1994). IEEE Trans. Electron Dev., 41, 643. 

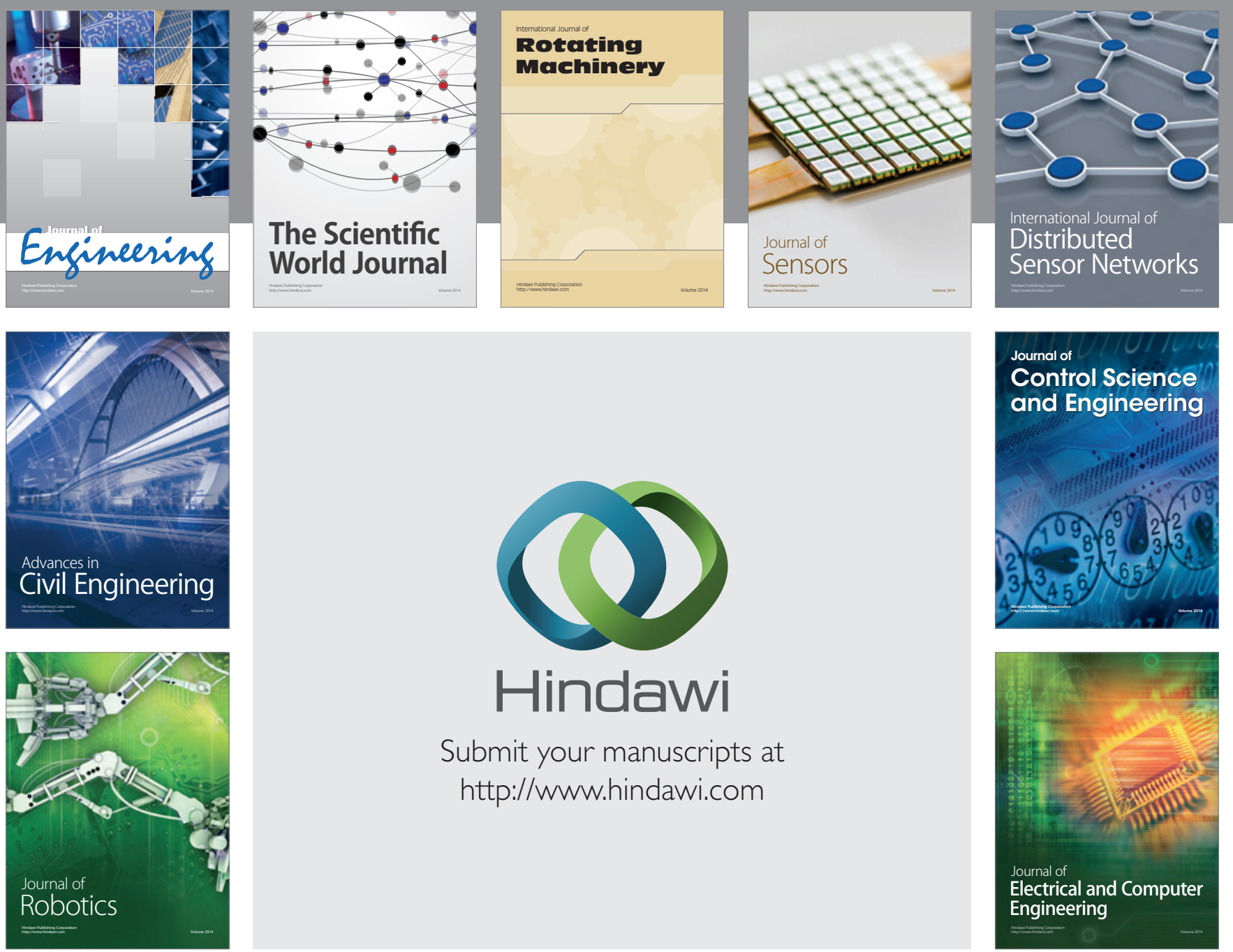

Submit your manuscripts at

http://www.hindawi.com
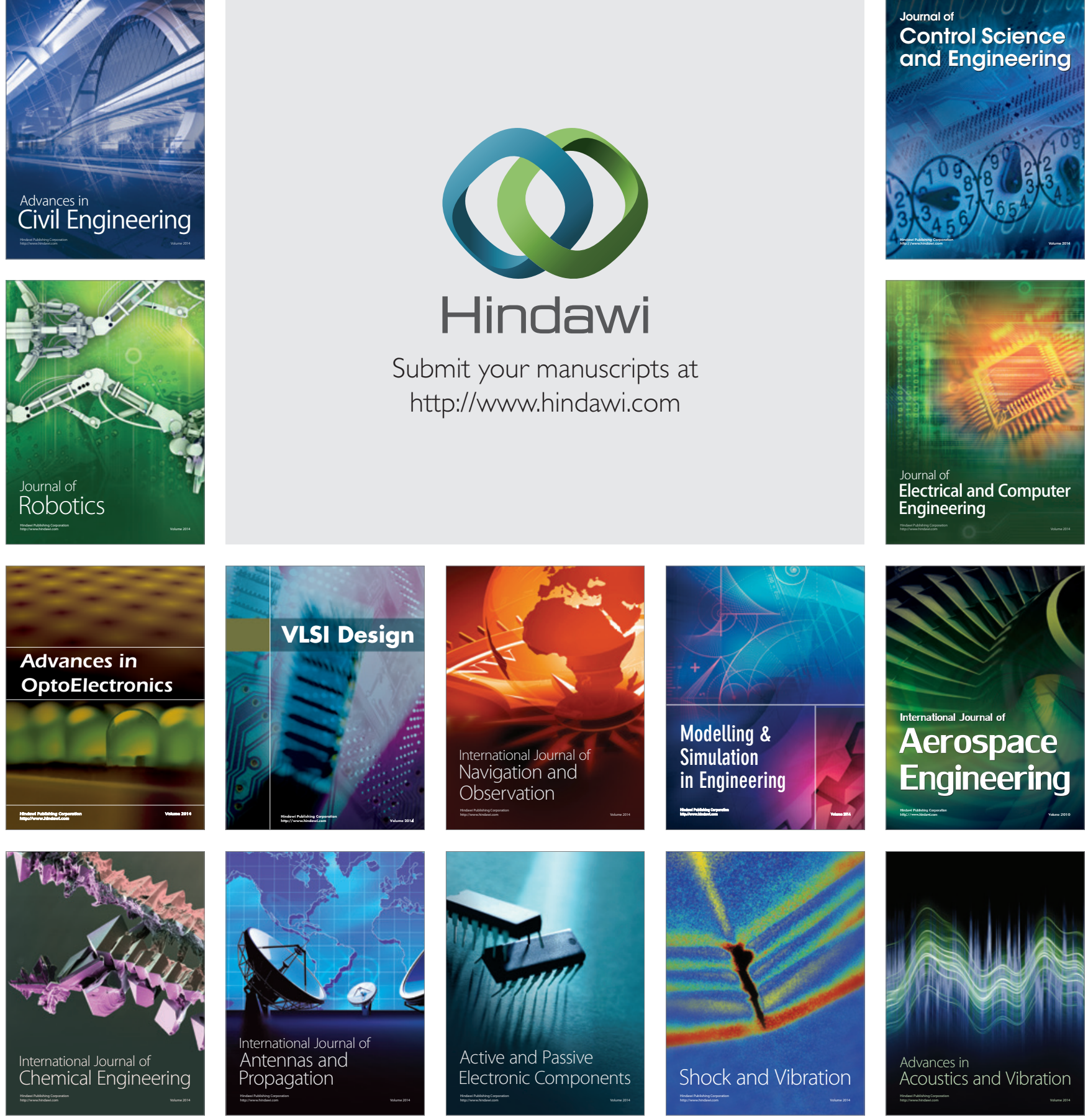